\title{
Proper autofocus for better particle measurements
}

\author{
M. Zs. Kiss ${ }^{\mathrm{ab}}$ \\ ${ }^{a}$ Computational Optical Sensing and Processing Laboratory, MTA SZTAKI, Kende utca 13-17., \\ Budapest, H-1111; ${ }^{\text {b}}$ Roska Tamás Doctoral School of Sciences and Technology, PPKE ITK, Práter \\ utca 50/a, Budapest, H-1083
}

\begin{abstract}
Digital holographic imaging is a good tool for automatic particle size and morphology measurements. One of its benefits is that flowing samples can be observed, but the focus - the reconstruction distance- should be well defined automatically to reach the best quality image reconstruction during real time measurements.

Now, we present a new way to increase the accuracy of the autofocus. It is not another autofocus algorithm, but it can be used with different autofocus algorithms. Our idea was to change the input images of the autofocus algorithm. The theoretical foundation of this modification is the known connection between the numerical aperture and the depth of field. A focused target beam contains central rays with smaller and extremal rays with higher converging angles. Imaging without the central rays, the rays that increase the depth of focus will be missing but the rays which carry the details will be kept. Using only the extremal rays at the imaging we can see more clearly whether the image is in focus or not, even though it has missing information. Therefore, we reconstructed the input images of the autofocus algorithm from the hologram without the central rays. The reconstruction was made with the angular spectrum method. The central rays were eliminated with a proper frequency-filtering of Fourier transform of the hologram.

This new method was tested with different autofocus algorithms, and its efficiency can be clearly observed. We used it in an in-line holographic setup, which was built to observe algae from algae cultures.
\end{abstract}

Keywords: digital holography, autofocus, microscopy, real time measurements

\section{INTRODUCTION}

In optical imaging to find the appropriate focus is a main point. Using the proper focus the resolution, accuracy and quality of the image can be maximized to have the finest measurements and better visual experiences. The determination of the proper focus can be achieved in different ways. Usually it is set empirically by a human operator, but it is not the way of automatization. To define the focus distance, with tools and methods physical measurements can be made e.g. with the wedge prism, micro-prisms [1], knife edge method [2], phase differences measurement with a dual sensor, and other kind of distance measurements. The most frequently applied methods are comparing the images that belong to different focal distances. These image-analysis-based focusing methods are the most common ones in automata, digital and numerical measurements. They give a focal metric to every images (that belong to the same object and) that are connected to different depths. The focal metric is a scalar value. The image that's focal metric is an extremum is in focus by the used focus measurement algorithm. Usually sharpness [3] or contrast [4] is measured. In the literature there are many kind of solutions, and there are many articles that are comparing $[5,6]$ them. For different problems different focus measure algorithms are used.

Our aim was to have a better autofocus result to have a proper focus distance. This is needed for our in-line [7] digital holographic microscope that captures hologram instead of images. With our approach we observe flowing samples that contain many point-like objects which are algae. From a captured hologram we can numerically reconstruct the images of the different objects that are in different depths.

Our idea was to modify the input images of the autofocus algorithm instead of the algorithm to have a better autofocus result. In the case of holography we apply numerical wave field propagation. It is known that rays connected to a higher numerical aperture have higher divergence angle. So we tested that if imaging is done only with these rays the accuracy of the focus distance measurement can be increased or not. We have to emphasize that these modified images are used only to determine the proper focus distance of an object. The original hologram and the proper focus distance are used to reconstruct the full image of an object for sample analyzes. 
In this paper first we present the theoretical physical background of our idea. Next we present our digital holographic setup that captures the hologram and the modification of the image reconstruction for focus measurement. Finally the results of the image modification, the effect of the image modification on twenty-six different autofocus algorithms and the result of this implementation to our digital holographic microscope measurements are presented.

\section{THE THEORETICAL BACKGROUND}

The inputs of the numerical autofocus methods are the images of an object of different image planes around the focus. Every image is characterized by a focus metric. Where this metric has a minimum or maximum, there is the focused image plane. The other image planes are out of focus. When the focus measure algorithm creates large extremal, the result can be found more easily.

Using holographic imaging the light propagation from the hologram to the image planes is handled. It means that with the modification of the reconstruction, there is another way to make a new kind of input images for the focus measure algorithms. We can get different kind of input images with the reconstruction of a captured hologram than we can get form a modified captured image. Holography uses complex numbers while captured images are scalar. Below we present why and how to change the light propagation to modify the input images to get more precise focus measurement.

\subsection{Optical consideration}

First let observe an imaging using ray optics. Figure 1. a)-b) shows that if the numerical aperture (NA) is increased then the depth of field $(\delta)$ is decreased. They are in inverse proportion: $\delta \approx \frac{1}{N A}$. The numerical aperture is defined by the extremal rays diverging half angle $(\theta) . N A=n * \sin (\theta)$. So drawing extremal rays the focus point can be pointed out more clearly as it can be seen compering Figure 1. a) to c). Leaving the focal point the extremal rays will spread more than the central rays. It is also known that the waist of a beam is smaller when the numerical aperture is bigger.
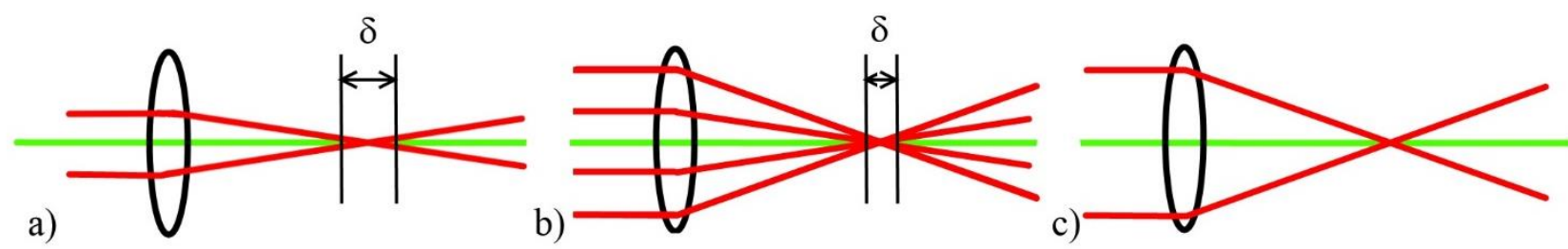

Figure 1. a)-b) The connection between the numerical aperture (NA) and the depth of field. c) Cutting out the central rays, the effect of the extremal rays can be more dominant.

Modifying some of the parameters of the propagation such as wavelength, during the propagation the numerical aperture can be increased, but it is not a good solution because the reconstruction distances will also change in a wrong way. Because there is no way to increase the numerical aperture we decided to make the extremal rays more dominant, so we filter out the central rays that have usually higher intensity also as it can be seen in Figure 1. c). Without the central rays the image biased considerably, but the difference and so the contrast between the in focus and out of focus images will be bigger. Imaging without the central rays is imaging with a gappy wavefront.

There are examples in astronomy [8] where they increas the numerical aperture to have better resolution although they get a gappy electromagnetic wavefront. At holography we can also get an image from a gappy hologram [9] which is a captured gappy wavefront.

We want to mention here that there are aberrations that can considerably modify the results. Holography is a monochrome imaging technique, so chromatic aberration does not play role, but spherical aberration (and coma) can appear. Spherical aberration as Figure 2. shows became by focusing a beam to different distances. It is caused by the lens and it is a function of the distance from the optical axis to the ray where it hit the lens. This can make an offset when we measur the focus 
using dominantly the extremal rays. Considering it from another point of view, this filtered hologram propagation can be applied for measuring spherical aberration of the optical setup.

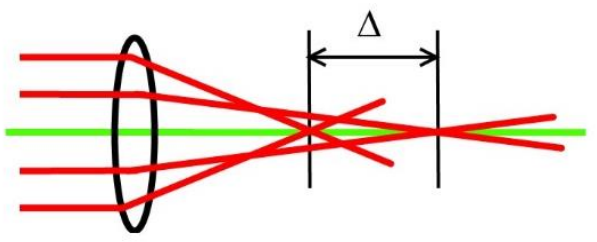

Figure 2. The spherical aberration can make an offset $(\Delta)$ at the focus measurement.

\subsection{Holographic implementation}

We used holography for our measurements. The object's wavefront is captured as a thin digital hologram. We used angular spectrum method [10] to emulate the propagation of the wave field. The used reconstruction distances are small and this method does not use any approximation. The reference and the reconstruction beams are both plane waves. In this setup the wave field propagation is a hologram convolution with a spherical wave field. The convolution is a multiplication in frequency space, so the Fourier transform of the hologram is multiplied by the transfer function. Inverse Fourier transforming the result, we get the reconstructed image.

To make the reconstruction using only the extremal rays we can cut out the middle of the hologram of every object or also we can make a low frequency filtering. We chose the second solution. It is easier, and making on the full captured hologram it will done on every holograms of the objects. Frequency filtering is also done in the frequency space, so it can be carried out during the propagation.

The filtered reconstructions are the new input images. From them the focus algorithm creates their focus metrics. Where one of the extrema of the curve of the focus metrics is, there is the required focus distance. Figure 3 . is a flow image of the numerical method from the captured hologram to the decision, where the focuses of the objects are.

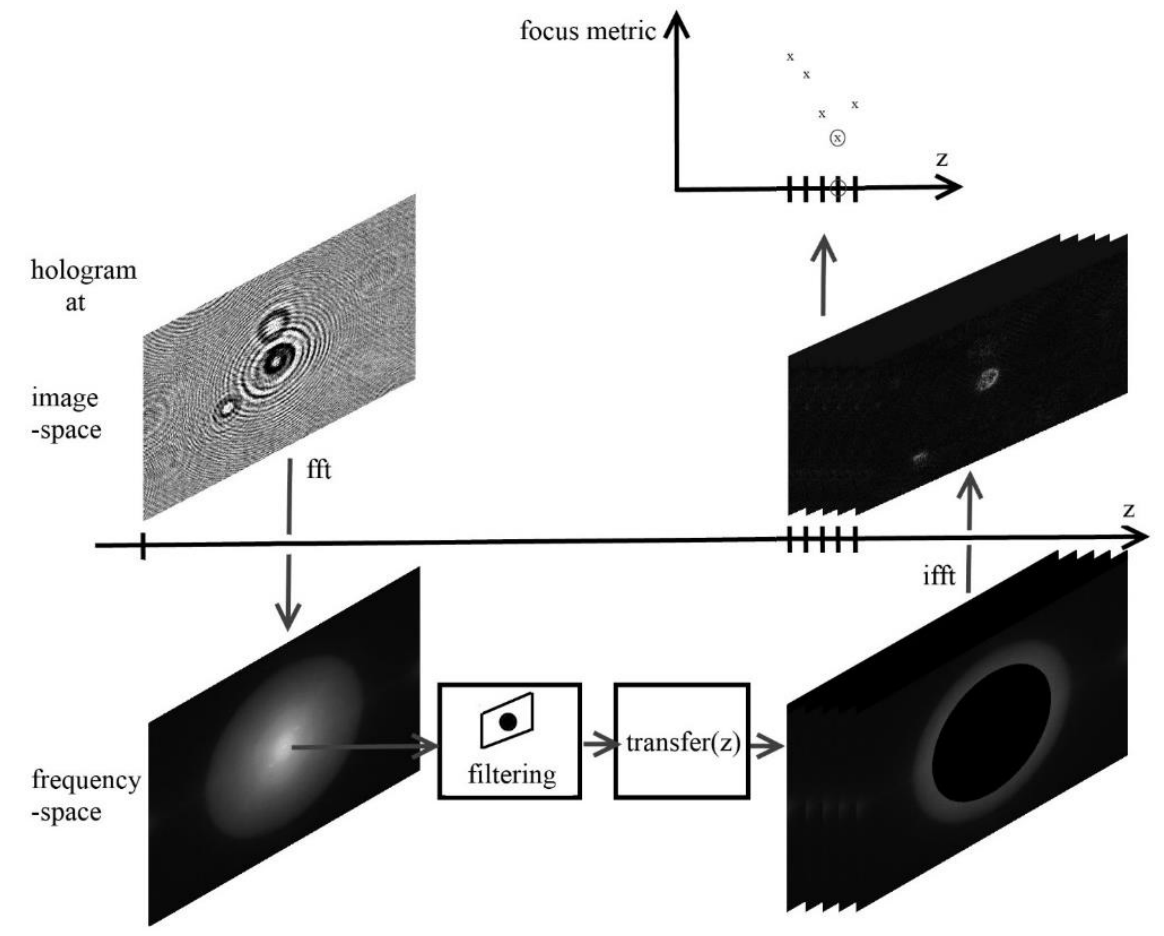

Figure 3. This is the flow image of the numerical method from the captured hologram to the decision, where the focuses of the objects are. 


\subsubsection{The size of the frequency filter}

Increasing the size of the frequency filter we can observe that the image intensity maximum getting darker but on the other hand the divergence became more visible, and also the standard deviation of the results of the twenty-six focus measure algorithms decrease. In Figure 4. the effect of the filter size on the divergence visibility can be seen. Observing the results of the standard deviation the best filter size was 0.3 times of the image diameter.

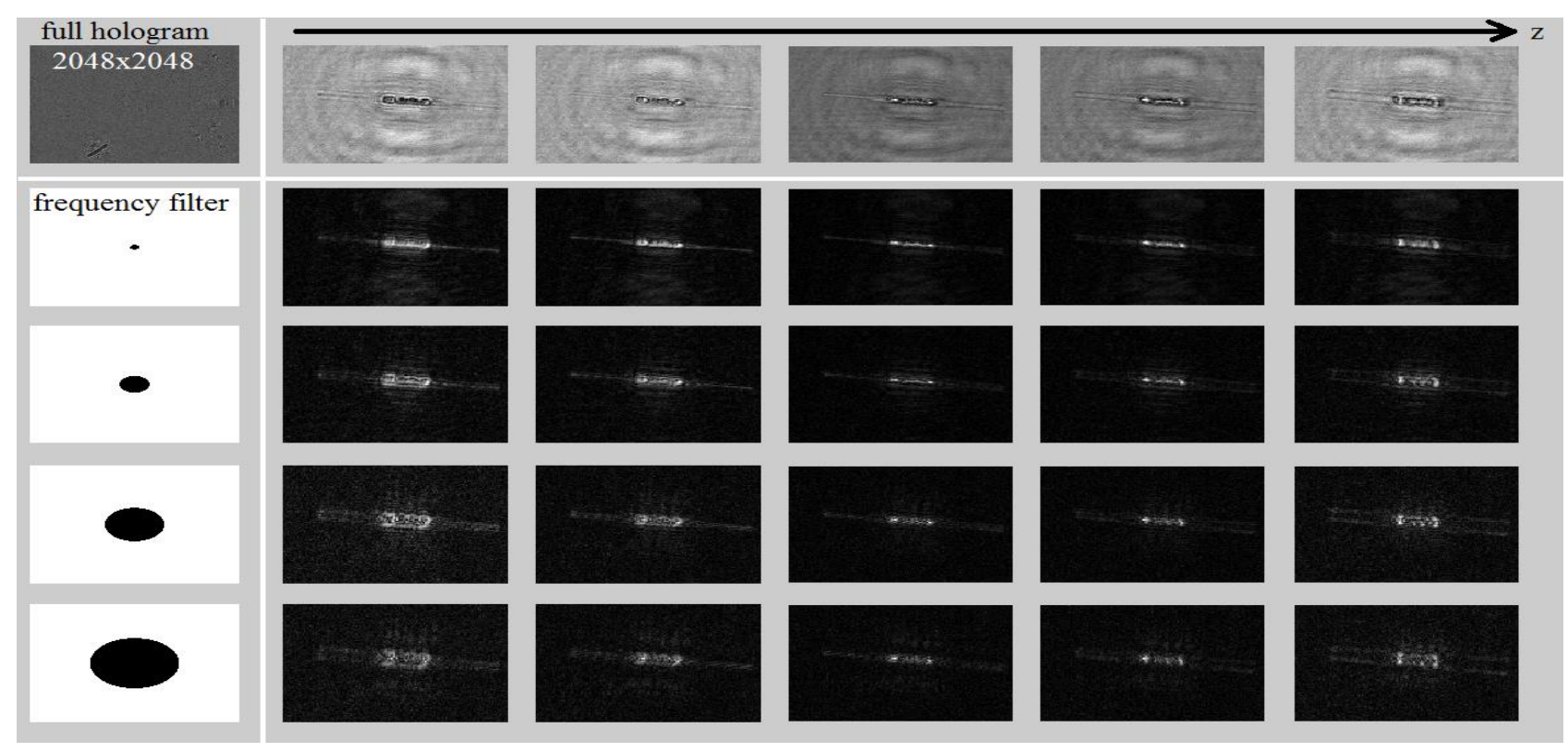

Figure 4. The effect of the filter size on the visibility of the divergence is shown here with an object reconstruction.

\section{OPTICAL SETUP}

The optical setup is an in-line holographic microscope [11], built for flowing sample measurements. That is why the sample has to be rare and small objects. We measure algae with the setup. The monochrome camera (EPIX sv10m6) captures hologram with a wavelength of $473 \mathrm{~nm}$. An afocal [12] optical system is built in front of the camera to magnify and fit the hologram to the detector. It contains an Olympus objective (20x LUCPLFLN) that has a focal length of $9 \mathrm{~mm}$ and an achromatic tube lens with a focal length of $60 \mathrm{~mm}$. The detector is in the back focal plane of the tube lens, and the closest plane of the flowing cell is in the front focal plane of the objective. So the objects are out of focus. The thickness of the flowing cell is $0.8 \mathrm{~mm}$. The lateral magnification of the optical setup is $6,7 \mathrm{x}$ and the longitudinal magnification is $44,4 \mathrm{x}$. The light source is a single mode fiber coupled laser $12 \mathrm{~cm}$ far from the flowing cell. Figure 5 . show the optical setup.
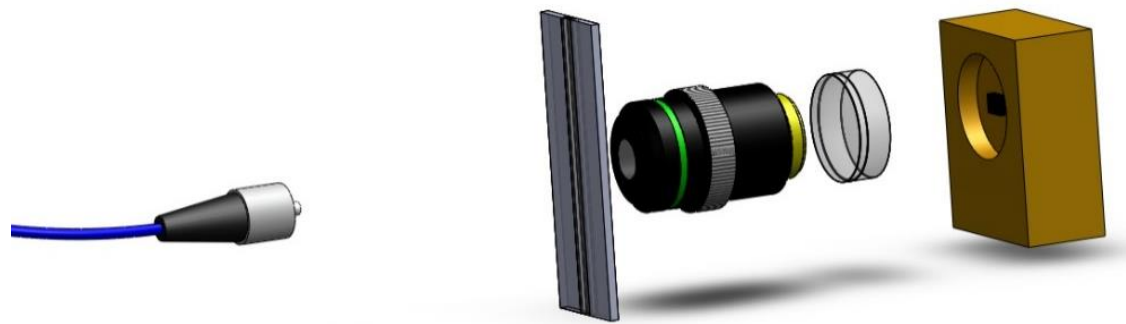

Figure 5. The optical setup of the in-line monochrome digital holographic microscope. From left to right there is the fiber coupled single mode blue laser with $473 \mathrm{~nm}$ wavelength, the flowing cell with a depth of $0.8 \mathrm{~mm}$, the Olympus objective (20x LUCPLFLN) and an achromatic tube lens ( $\mathrm{f}=60 \mathrm{~mm})$ as an afocal system and the EPIX (sv10m6) digital monochrome camera. 


\section{MEASUREMENTS}

The holograms are measured with the presented DHM setup. From the captured hologram we generated the normal and the modified input images for the focus measure algorithms. We present the effects of the modification using the normal input as a reference. We used 26 different focus measure algorithms for testing the applicability of the modified input image method. The numeric algorithm was written in MATLAB. The used operators for the focus measure algorithms were available in a package and its basic is the "Analysis of focus measure operators for shape-from-focus" [13] article. Below in Table 1. we present the used autofocus operators. Our, the twenty-seventh focus measure algorithm that is built into the DHM is a mixed algorithm. It measures contrast and sharpness equally.

Table 1. Twenty-six focus measure operators that are used here to test the effects of the modification of the input images.

\begin{tabular}{|c|c|c|}
\hline $\begin{array}{l}\text { Operator name in } \\
\text { MATLAB }\end{array}$ & $\begin{array}{l}\text { Operator name in the } \\
\text { literature }\end{array}$ & Citation \\
\hline 'ACMO' & Absolute Central Moment & (Shirvaikar2004) [14] \\
\hline 'BREN' & Brenner's & (Santos97) [15] \\
\hline 'CONT' & Image contrast & (Nanda2001) [16] \\
\hline 'CURV' & Image Curvature & (Helmli2001) [17] \\
\hline 'DCTE' & Discrete cosine transform (DCT) energy ratio & (Shen2006) [18] \\
\hline 'GDER' & Gaussian derivative & (Geusebroek2000) [19] \\
\hline 'GLVA' & Gray level variance (GLV) & (Krotkov86) [20] \\
\hline 'GLLV' & Gray level local variance & (Pech2000) [21] \\
\hline 'GLVN' & Normalized gray level variance & (Santos97) [15] \\
\hline 'GRAE' & Energy of gradient & (Subbarao92a) [22] \\
\hline 'GRAT' & Threshold gradient & (Santos97) [15] \\
\hline 'GRAS' & Squared gradient & (Eskicioglu95) [23] \\
\hline 'HELM' & Helmli's mean method & (Helmli2001) [17] \\
\hline 'HISE' & Histogram entropy & (Krotkov86) [20] \\
\hline 'LAPE' & Energy of Laplacian & (Subbarao92a) [22] \\
\hline 'LAPM' & Modified Laplacian & (Nayar90) [24] \\
\hline 'LAPV' & Variance of Laplacian & (Pech2000) [21] \\
\hline 'LAPD' & Diagonal Laplacian & (Thelen2009) [25] \\
\hline 'SFIL' & Steerable filters & (Minhas2009) \\
\hline 'SFRQ' & Spatial frequency & (Eskicioglu95) [23] \\
\hline 'TENG' & Tenengrad & (Krotkov86) [20] \\
\hline 'TENV' & Tenengrad variance & (Pech2000) [21] \\
\hline 'VOLA' & Vollath's correlation & (Santos97) [15] \\
\hline 'WAVS' & Sum of Wavelet coefficients & (Yang2003) [26] \\
\hline 'WAVV' & Variance of wavelet coefficients & (Yang2003) [26] \\
\hline 'WAVR' & Ratio of wavelet coefficients & (Xie2006) [27] \\
\hline
\end{tabular}




\subsection{Divergence and contrast}

The first observation on the input image modification is that not only the input images got a higher contrast, but the obtained focus metric results. This is shown in Figure 6. In that figure an alga is reconstructed in the mentioned two ways. The gray image line presents the original reconstruction and the line with black background presents the modified reconstructions. It can be seen that without the low frequency components the background became dark. It can also be seen well that the diverging rays make easier to see, where the focus is. The points of the green and the blue curves are the calculated focus metrics. 'ACMO' operator was used. The blue (original) result is not constant but has a smaller contrast than the green curve has. Later, to become able to compare these results, we will normalize them between [0 1], and we will mirror the curves -where it is needed- to use in every result the minimum point to determine the focus distance.

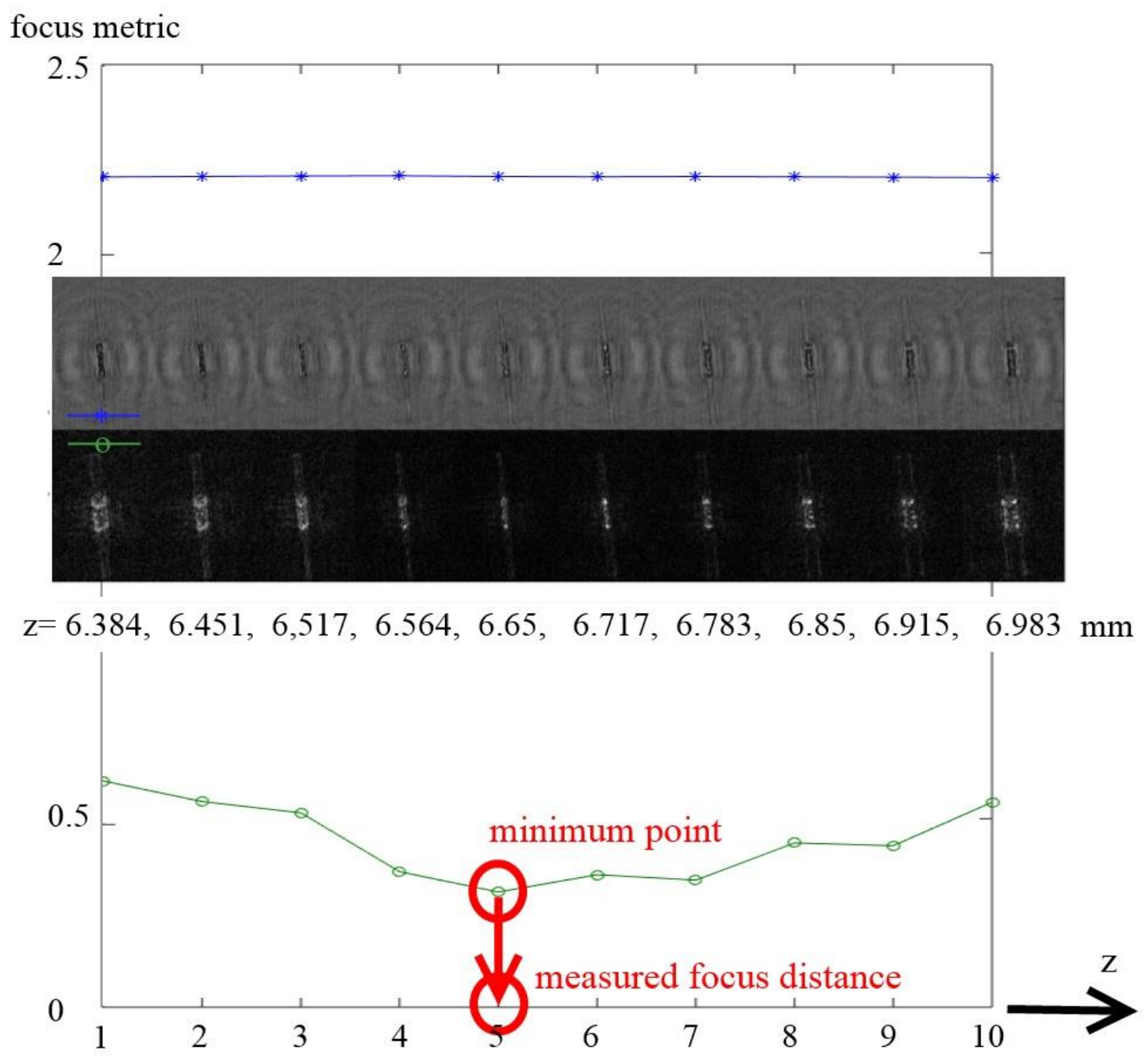

Figure 6. The gray image line presents the original reconstruction and the line with black background presents the modified reconstructions. It can be seen that without the low frequency components the background became dark. It can also be seen well that the diverging rays makes easier to see, where the focus is. The points of the green and the blue curves are the calculated focus metrics. 'ACMO' operator was used.

Using in-line holography after reconstruction twin image is on and around the image of the object. Making the low frequency filtering the twin image won't disturb the reconstructed image with its interference fringes. This advantage clearly can be seen. 


\subsection{Testing the modified images on 26 autofocus algorithms}

The test target to measure the applicability of the modified input images method was a round shaped green alga. It is both a phase and intensity target. Twenty-six focus measure algorithms described the focus distance using original (up) and modified (down) reconstructions. Using the modified inputs, the twenty-six results has a smaller standard deviation. It can be seen in Figure 7.
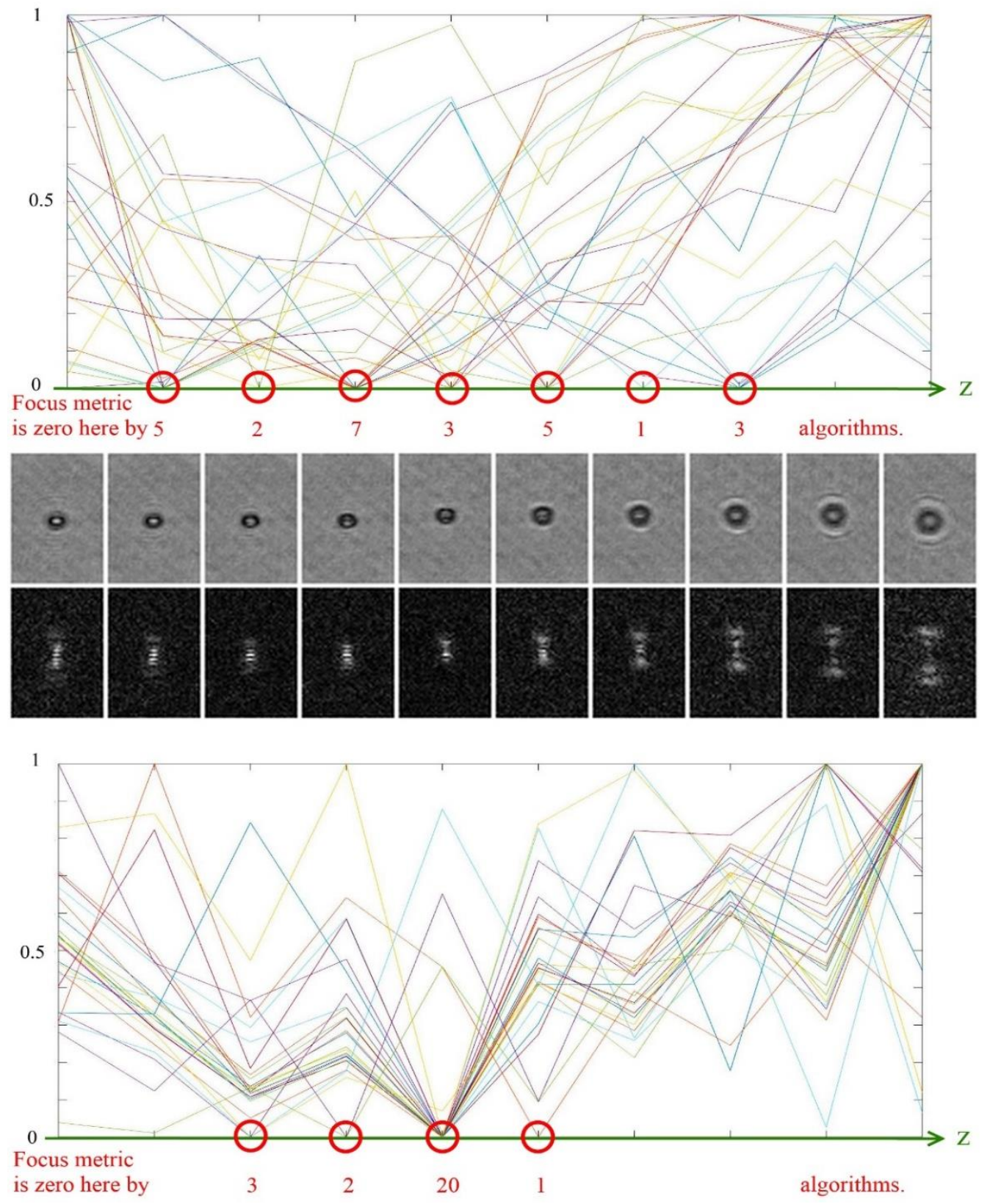

Figure 7. Twenty-six focus measure algorithms calculated the focus distance using original (up) and modified (down) reconstructions. Using the modified inputs, the twenty-six results has a smaller standard deviation.

It means, that the modified input images point out more clearly the real focus distance. 


\subsection{Implementing into the setup}

We implemented this modified image method into our digital holographic microscope. Only the object reconstruction for autofocus measurement was modified. The autofocus algorithm and the image reconstruction for sample analyzing were not. In this measurement we pointed out two holograms and measured 25-25 objects per each. We defined their focus distance by human, with the old measuring method and with the modified one. We compared the two automata results, and we got that the new, modified method were 29 times closer to the result while the older only 21 times. The reconstructed images of the setup in Figure 8 can be seen.
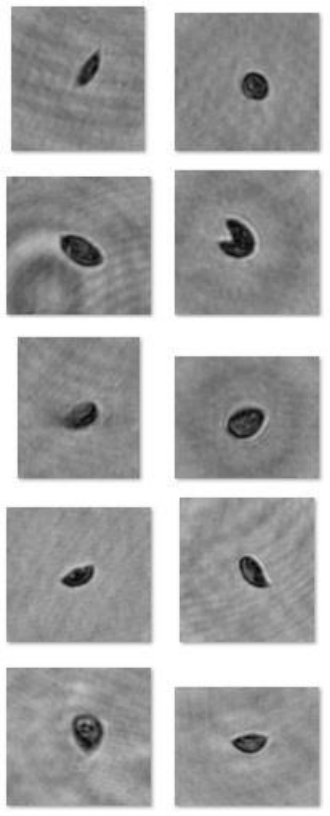


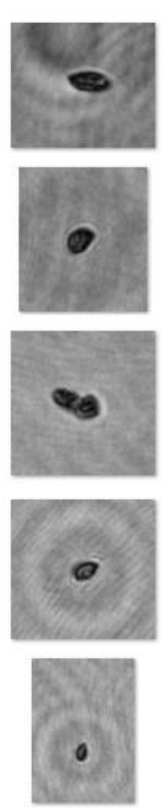


Figure 8 . The fifty reconstructed images from two holograms that were measured by our DHM setup. The sample was from a Scenedesmus algae culture.

\section{CONCLUSION}

Low frequency filtering a complex hologram and comparing its reconstructed images of the same objet having different reconstruction distances, it becomes more evident for human and for many of the focus measure algorithms that which one is in focus.

\section{REFERENCES}

[1] Chao, K.-S., Lin, M.-S., and Yang, R.-J., “An in-plane optofluidic microchip for focal point control,” Lab on a Chip 13(19), 3886-3892 (2013).

[2] Dorn, R., Quabis, S., and Leuchs, G., "Sharper focus for a radially polarized light beam," Phys. Rev. Lett. 91, 233901 (Dec 2003).

[3] Yousefi, S., Rahman, M., and Kehtarnavaz, N., “A new auto-focus sharpness function for digital and smartphone cameras," IEEE Transactions on Consumer Electronics 57(3) (2011).

[4] Gamadia, M., Kehtarnavaz, N., and Roberts-Hoffman, K., "Low-light auto-focus enhancement for digital and cell-phone camera image pipelines," IEEE Transactions on Consumer Electronics 53(2) (2007).

[5] Firestone, L., Cook, K., Culp, K., Talsania, N., and Preston Jr, K., "Comparison of autofocus methods for automated microscopy," Cytometry: The Journal of the International Society for Analytical Cytology 12(3), 195-206 (1991).

[6] Langehanenberg, P., Kemper, B., and von Bally, G., "Autofocus algorithms for digital-holographic microscopy," in [European Conference on Biomedical Optics0.1em], 6633_13, Optical Society of America (2007).

[7] Gabor, D., "Microscopy by reconstructed wave-fronts," Proceedings of the Royal Society of London. Series A, Mathematical and Physical Sciences 197(1051), pp. 454-487 (1949). 
[8] Ananthakrishnan, S., “Antennas for radio astronomy,” in [2009 Applied Electromagnetics Conference (AEMC)0.1em], 1-4 (Dec 2009).

[9] Kiss, M. Z., "Ring-shaped bifocal lens used for fluorescent self-referenced holographic imaging," Journal of the European Optical Society-Rapid Publications 12, 2 (Jun 2016).

[10] Matsushima, K. and Shimobaba, T., "Band-limited angular spectrum method for numerical simulation of freespace propagation in far and near fields," Opt. Express 17, 19662-19673 (Oct 2009).

[11] Kiss, M. Z., Nagy, B. J., Lakatos, P., Göröcs, Z., Tőkés, S., Wittner, B., and Orzó, L., "Special multicolor illumination and numerical tilt correction in volumetric digital holographic microscopy," Opt. Express 22, 7559-7573 (Apr 2014).

[12] Tőkés, S. and Orzó, L., "Afocal digital holographic microscopy and its advantages," in [2012 13th International Workshop on Cellular Nanoscale Networks and their Applications0.1em], 1-5 (Aug 2012).

[13] Pertuz, S., Puig, D., and Garcia, M. A., "Analysis of focus measure operators for shape-from-focus," Pattern Recognition 46(5), 1415 - 1432 (2013).

[14] Shirvaikar, M. V., "An optimal measure for camera focus and exposure," in [Thirty-Sixth Southeastern Symposium on System Theory, 2004. Proceedings of the0.1em], 472-475 (March 2004).

[15] Brenner, J. F., Dew, B. S., Horton, J. B., King, T., Neurath, P. W., and Selles, W. D., “An automated microscope for cytologic research a preliminary evaluation.," Journal of Histochemistry \& Cytochemistry 24(1), 100 111 (1976). PMID: 1254907.

[16] Nanda, H. and Cutler, R., "Practical calibrations for a real-time digital omnidirectional camera," CVPR Technical Sketch 20, 2 (2001).

[17] Helmli, F. S. and Scherer, S., "Adaptive shape from focus with an error estimation in light microscopy," in [ISPA 2001. Proceedings of the 2nd International Symposium on Image and Signal Processing and Analysis. In conjunction with 23rd International Conference on Information Technology Interfaces (IEEE Cat.0.1em], 188-193 (June 2001).

[18] Shen, C.-H. and Chen, H. H., "Robust focus measure for low-contrast images," in [2006 Digest of Technical Papers International Conference on Consumer Electronics0.1em], 69-70 (Jan 2006).

[19] Geusebroek, J.-M., Cornelissen, F., Smeulders, A. W., and Geerts, H., "Robust autofocusing in microscopy," Cytometry: The Journal of the International Society for Analytical Cytology 39(1), 1-9 (2000).

[20] Krotkov, E. and Martin, J. ., "Range from focus," in [Proceedings. 1986 IEEE International Conference on Robotics and Automation0.1em], 3, 1093-1098 (April 1986).

[21] Pech-Pacheco, J. L., Cristobal, G., Chamorro-Martinez, J., and Fernandez-Valdivia, J., "Diatom autofocusing in brightfield microscopy: a comparative study," in [Proceedings 15th International Conference on Pattern Recognition. ICPR-20000.1em], 3, 314-317 vol.3 (Sep. 2000).

[22] Murali Subbarao, Tae-Sun Choi, A. N., "Focusing techniques," Optical Engineering 32, 32 - 32 - 13 (1993)

[23] Eskicioglu, A. M. and Fisher, P. S., "Image quality measures and their performance," IEEE Transactions on Communications 43, 2959-2965 (Dec 1995).

[24] Nayar, S. K. and Nakagawa, Y., "Shape from focus: an effective approach for rough surfaces," in [Proceedings., IEEE International Conference on Robotics and Automation0.1em], 218-225 vol.2 (May 1990).

[25] Thelen, A., Frey, S., Hirsch, S., and Hering, P., "Improvements in shape-from-focus for holographic reconstructions with regard to focus operators, neighborhood-size, and height value interpolation," IEEE Transactions on Image Processing 18, 151-157 (Jan 2009).

[26] Yang, G. and Nelson, B. J., "Wavelet-based autofocusing and unsupervised segmentation of microscopic images," in [Proceedings 2003 IEEE/RSJ International Conference on Intelligent Robots and Systems (IROS 2003) (Cat. No.03CH37453)0.1em], 3, 2143-2148 vol.3 (Oct 2003).

[27] Xie, H., Rong, W., and Sun, L., "Wavelet-based focus measure and 3-d surface reconstruction method for microscopy images," in [2006 IEEE/RSJ International Conference on Intelligent Robots and Systems0.1em], 229-234 (Oct 2006). 